COELHO, MFB; AZEVEDO, RAB. 2016. Efeito do tipo de estaca na propagação de Turnera subulata. Horticultura Brasileira 34:435-438. DOI - http:// dx.doi.org/10.1590/S0102-05362016003021

\title{
Efeito do tipo de estaca na propagação de Turnera subulata
}

\author{
Maria FB Coelho ${ }^{1}$; Rodrigo AB Azevedo ${ }^{2}$ \\ ${ }^{1}$ Universidade Federal de Mato Grosso (UFMT), Cuiabá-MT, Brasil; coelhomfstrela@gmail.com ²Universidade da Integração \\ Internacional da Lusofonia Afro Brasileira (UNILAB), Redenção-CE, Brasil; rodrigo.abazevedo@gmail.com
}

\section{RESUMO}

Turnera subulata é uma espécie herbácea e ruderal, conhecida no Brasil como Chanana, Damiana ou Flor-do-Guarujá e tem amplo uso como ornamental e na medicina popular o que torna a espécie importante para o cultivo. O objetivo do presente trabalho foi avaliar o efeito da posição da estaca na planta e presença ou ausência de folhas na propagação de T. subulata. No primeiro experimento foram avaliados os tratamentos a) estaca mediana com uma gema e sem folhas, b) com uma gema e duas folhas, c) com duas gemas e sem folhas e d) com duas gemas e duas folhas. No segundo experimento foram avaliadas estacas da posição apical, mediana e basal combinadas com presença e ausência de folhas. Foram avaliados o comprimento e número de raízes, número de folhas, massa seca de raízes, porcentagem de enraizamento e de sobrevivência. As estacas com duas gemas e duas folhas apresentaram maior comprimento de raiz $(10,5 \mathrm{~cm})$, número de raízes $(18,5)$, número de folhas $(6,6)$, massa seca da raiz $(0,48 \mathrm{mg})$ e porcentagem de enraizamento $(90 \%)$ e de sobrevivência (100\%). As estacas basais com folhas apresentaram maior comprimento da raiz $(10,5 \mathrm{~cm})$, número de raízes $(18,5)$ e de folhas $(6,6)$, maior porcentagem de enraizamento $(96,8 \%)$ e de sobrevivência (100\%). Estacas com uma gema e estacas apicais apresentaram menor porcentagem de sobrevivência (em torno de $70 \%$ ). Para a propagação de T. subulata recomenda-se o uso de estacas basais com duas folhas.

\section{ABSTRACT}

\section{Cutting type for the Turnera subulata propagation}

Turnera subulata is an herbaceous and ruderal species, known in Brazil as Chanana, Damiana or Flor-do-Guarujá widely used as an ornamental plant as well as in folk medicine. The aim of this study was to evaluate the effect of cutting position on the plant and the presence or absence of leaves in the T. subulata propagation. In the first experiment the treatments were a) median cutting with a bud and without leaves, b) with a bud and two leaves, c) with two buds and without leaves and d) with two buds and two leaves. In the second experiment we evaluated apical, median and basal cuttings combined with leaves and without leaves. We evaluated length and number of roots, number of leaves, dry matter of roots, rooting and survival. The cuttings with two buds and two leaves had higher root length $(10.5 \mathrm{~cm})$, number of roots (18.5), number of leaves (6.6), dry mass of roots $(0.48 \mathrm{mg})$, percentage of rooting $(90 \%)$ and survival $(100 \%)$. The basal cuttings with leaves had higher root length $(10.5$ $\mathrm{cm})$, number of roots (18.5) and leaves (6.6), the highest percentage of rooting $(96.8 \%)$ and survival $(100 \%)$. Cuttings with one bud and apical cuttings have a lower survival rate, around $70 \%$. For the $T$. subulata propagation we recommend the use of basal cuttings with two leaves.

Keywords: Chanana, rooting, cutting.

Palavras-chave: Chanana, enraizamento, estaquia.

(Recebido para publicação em 5 de abril de 2015; aceito em 5 de março de 2016) (Received on April 5, 2015; accepted on March 5, 2016)

A família Turneraceae compreende 10 gêneros e 226 espécies, sendo a maioria distribuída nos Neotrópicos (Shore et al., 2006). O gênero Turnera apresenta aproximadamente 128 espécies, das quais quatro (Turnera oculata, Turnera thomasii, Turnera ulmifolia e Turnera subulata) foram introduzidas e se espalharam em várias regiões neotropicais do mundo (Arbo, 2005).

Turnera subulata é herbácea e ruderal, conhecida no Brasil como Chanana, Damiana ou Flor-do-Guarujá e as populações ocorrem nas margens das estradas e em áreas associadas com algum distúrbio antrópico (Arbo, 2005). Turnera subulata é utilizada contra amenorréia na forma de chá (Agra et al., 2007).

As espécies do gênero Turnera têm amplo uso ornamental e as raízes e as folhas são empregadas na medicina popular como expectorante contra tosse, gripe, bronquite, inflamações, problemas de próstata e câncer (Agra et al., 2007; Albuquerque et al., 2007). Algumas atividades farmacológicas foram comprovadas em espécies desse gênero, como anti-inflamatória, antiulcerogênica (Galvez, 2006), antioxidante (Nascimento et al., 2006) e antimalárica (Antoun et al., 2001) e moluscida (Santos et al., 2010).

A presença de heterostilia em flores de Turnera subulata (Schlindwein \&
Medeiros, 2006) ocasiona a polinização cruzada e assim as sementes de um mesmo individuo podem ter diferentes genótipos. A estaquia é uma das formas de propagação vegetativa que mantem as características dos indivíduos selecionados e tem como vantagem a abundância de materiais propagativos. Assim, o objetivo no presente trabalho foi verificar o efeito do tipo de estaca na propagação de T. subulata.

\section{MATERIAL E MÉTODOS}

Os experimentos foram conduzidos em casa telada no campus da Univer- 
sidade da Integração Internacional da Lusofonia Afro Brasileira (UNILAB), Redenção-CE. As estacas de Turnera subulata foram obtidas em 50 indivíduos em Acarape-CE.

Foram realizados dois experimentos. No primeiro foi usado o delineamento inteiramente casualizado com quatro tratamentos e cinco repetições com dez estacas. Os tratamentos foram estacas medianas, variando o número de gemas e a presença de folhas: a) com uma gema e sem folhas; b) com uma gema e com folhas; c) com duas gemas e sem folhas; e d) com duas gemas e com folhas. $\mathrm{O}$ segundo experimento foi inteiramente casualizado no esquema fatorial $3 \times 2$, com três tipos de estaca de $5 \mathrm{~cm}$ (apical, mediana e basal) combinadas com presença de folhas e ausência de folhas com cinco repetições de dez estacas.

Nos dois experimentos as estacas foram colocadas em bandejas de polietileno de 50 células preenchidas com substrato areia lavada e esterilizada. O substrato foi umedecido diariamente com duas irrigações manuais.

As características avaliadas aos 30 dias foram comprimento e número de raízes, número de folhas completamente desenvolvidas, massa seca de raízes, porcentagem de enraizamento (proporção de estacas com raízes em relação ao total da parcela) e de sobrevivência (proporção de estacas vivas em relação ao total da parcela) de estacas. Os dados foram submetidos à análise de variância pelo teste $\mathrm{F}$ e as médias comparadas pelo teste de Tukey $(\mathrm{p}<0,05)$.

\section{RESULTADOS E DISCUSSÃO}

No primeiro experimento houve diferença $(\mathrm{p} \leq 0,01)$ pelo teste $\mathrm{F}$ para todas as características avaliadas nas estacas de Turnera subulata, com os menores valores nas estacas com uma gema e sem folhas (Tabela 1). Estacas com uma gema e estacas apicais apresentaram menor porcentagem de sobrevivência, em torno de $70 \%$. As estacas com duas gemas e duas folhas apresentaram maior comprimento de raiz $(10,5 \mathrm{~cm})$, número de raízes $(18,5)$, número de folhas $(6,6)$, massa seca da raiz $(0,48$ $\mathrm{mg}$ ), porcentagem de enraizamento
(90\%) e de sobrevivência (100\%). Foi comprovado em outras espécies, como Olea europaea (Oliveira et al., 2003), Ficus carica (Pio et al., 2004), Ocimum gratissimum e Ocimum selloi (Purcino et al., 2012) e Rubus spp. (Vignolo et al., 2014) que a presença de folhas e gemas tem um papel-chave para a formação do novo sistema radicular.

A presença de folhas e gemas está relacionada com a produção de assimilados e de outras substâncias necessárias ao enraizamento das estacas. De acordo com Hartmann et al. (2011), há uma relação entre o enraizamento e o conteúdo de hidratos de carbono presentes nas estacas de algumas espécies, e a presença da folha, por ser fonte de auxina, é um importante fator na promoção do enraizamento de estacas de diversas espécies.

No segundo experimento houve diferença $(\mathrm{p} \leq 0,01)$ pelo teste $\mathrm{F}$ para o tipo de estaca, presença de folhas e interação entre tipo de estaca e presença de folhas para as características comprimento de raízes, porcentagem de enraizamento e de sobrevivência das estacas de $T$. subulata.

As estacas basais e medianas com folhas apresentaram maior comprimento da raiz $(10,5 \mathrm{~cm})$ e número de raízes (18,4 e 14,4 cm, respectivamente) que as estacas apicais $(5,6$ e 1,6 cm, respectivamente) (Tabela 2). Em várias estacas apicais observou-se podridão iniciando pela base. Estacas colhidas da posição apical do ramo têm células meristemáticas com metabolismo mais ativo, menor grau de lignificação e ausência ou menor quantidade de compostos fenólicos, o que facilita o enraizamento (Hartmann et al., 2011). Porém, baixos índices de enraizamento podem ocorrer como no presente estudo, devido à maior predisposição destas estacas tenras em perderem umidade.

As estacas medianas e basais com folhas apresentaram maior número de folhas (4,3 e 4,2 respectivamente) e massa seca de raiz $(0,38 \mathrm{mg})$ que as estacas apicais $(2,2$ e $0,20 \mathrm{mg}$ respectivamente) (Tabela 3), e também maior enraizamento e sobrevivência $(96,8$ e $100 \%$ respectivamente) (Tabela 4 ). A presença de folhas nas estacas basais foi importante para proporcionar maior enraizamento. De acordo com Hartmann et al. (2011), o efeito benéfico da presença das folhas em estacas é atribuído à produção de auxinas e cofatores, que são transportados para a base das estacas e pela continuação do processo da fotossíntese, responsável pela síntese de carboidratos necessários como fonte de energia para formação e crescimento das raízes.

Resultados semelhantes aos do presente estudo foram obtidos por Carvalho et al. (2015) que verificaram o maior número médio de raízes de Hyptis pectinata em estacas das posições mediana e basal e Albuquerque Junior et al. (2013) que verificaram que as estacas basais de Passiflora actínia, com a presença de folhas, proporcionaram o maior percentual de enraizamento, além de maior número de raízes, maior massa de matéria seca e maior comprimento

Tabela 1. Comprimento da raiz (CR), número de raízes (NR), número de folhas (NF), massa seca de raízes (MSR), porcentagem de enraizamento (PE) e de sobrevivência de estacas (PS) de Turnera subulata com diferentes numero de gemas e ausência ou presença de folhas root length (CR), number of roots (NR), number of leaves (NF), root dry mass (MSR), percentage of rooting (PE) and survival of cuttings (PS) of Turnera subulata with different number of buds and absence or presence of leaves\}. Redenção, UNILAB, 2013.

\begin{tabular}{|c|c|c|c|c|c|c|}
\hline \multirow{2}{*}{ Tipos de estaca } & \multirow{2}{*}{$\begin{array}{c}\mathrm{CR} \\
(\mathrm{cm})\end{array}$} & \multirow{2}{*}{ NR } & \multirow{2}{*}{ NF } & \multirow{2}{*}{$\begin{array}{r}\text { MSR } \\
(\mathrm{mg})\end{array}$} & $\mathbf{P E}$ & PS \\
\hline & & & & & \multicolumn{2}{|c|}{$(\%)$} \\
\hline uma gema e duas folhas & $14,5 \mathrm{a}$ & $12,7 \mathrm{~b}$ & $6,4 \mathrm{a}$ & $0,30 \mathrm{~b}$ & $55 \mathrm{c}$ & $60 \mathrm{c}$ \\
\hline uma gema e sem folhas & $5,7 \mathrm{c}$ & $1,8 \mathrm{c}$ & $2,2 \mathrm{c}$ & $0,20 \mathrm{c}$ & $50 \mathrm{c}$ & $60 \mathrm{c}$ \\
\hline duas gemas e duas folhas & $10,5 \mathrm{~b}$ & $18,5 \mathrm{a}$ & $6,6 \mathrm{a}$ & $0,48 \mathrm{a}$ & $90 \mathrm{a}$ & $100 \mathrm{a}$ \\
\hline duas gemas e sem folhas & $10,4 \mathrm{~b}$ & $15,1 \mathrm{ab}$ & $4,2 \mathrm{~b}$ & $0,20 \mathrm{c}$ & $70 \mathrm{~b}$ & $80 \mathrm{~b}$ \\
\hline $\mathrm{CV}(\%)$ & 22,5 & 24,4 & 15,6 & 12,3 & 12,7 & 12,9 \\
\hline
\end{tabular}

Letras minúsculas dentro da coluna comparam as medias para cada característica (lowercase

letters compare the averages within the column for each characteristic). 
Tabela 2. Comprimento e número de raízes de estacas de Turnera subulata obtidas em diferentes posições no ramo da planta e com ausência ou presença de folhas (length and number of roots in cuttings of Turnera subulata obtained at different positions in the plant and with presence or absence of leaves). Redenção, UNILAB, 2013.

\begin{tabular}{lcccccc}
\hline \multirow{2}{*}{ Tipo de Estaca } & \multicolumn{2}{c}{ Comprimento da raiz $(\mathbf{c m})$} & & \multicolumn{2}{c}{ Número de raízes } \\
\cline { 2 - 3 } \cline { 5 - 6 } & Ausência de folhas & Presença de folhas & & Ausência de folhas & Presença de folhas \\
\hline Apical & $6,45 \mathrm{aB}$ & $5,67 \mathrm{aB}$ & $1,95 \mathrm{aB}$ & $1,67 \mathrm{aB}$ \\
Mediana & $10,00 \mathrm{bA}$ & $14,50 \mathrm{aA}$ & & $12,72 \mathrm{bA}$ & $14,42 \mathrm{aA}$ \\
Basal & $5,37 \mathrm{bB}$ & $10,55 \mathrm{aA}$ & & $10,12 \mathrm{aA}$ & $18,47 \mathrm{bA}$ \\
\hline
\end{tabular}

Letras minúsculas comparam as médias dentro da linha e as maiúsculas dentro da coluna para cada característica (lowercase letters compare the averages within the line and capital letters within the column for each characteristic).

Tabela 3. Número de folhas e massa seca de raízes de estacas de Turnera subulata obtidas em diferentes posições no ramo da planta e com ausência ou presença de folhas (number of leaves and dry weight of root from cuttings of Turnera subulata obtained at different positions in the plant and with presence or absence of leaves). Redenção, UNILAB, 2013.

\begin{tabular}{lcccccc}
\hline \multirow{2}{*}{ Tipo de Estaca } & \multicolumn{2}{c}{ Número de folhas } & & \multicolumn{2}{c}{ Massa seca de raízes (mg) } \\
\cline { 2 - 3 } \cline { 5 - 7 } & & Ausência de folhas & Presença de folhas & & Ausência de folhas & Presença de folhas \\
\hline Apical & $2,45 \mathrm{aB}$ & $2,25 \mathrm{aB}$ & & $0,30 \mathrm{aAB}$ & $0,20 \mathrm{bB}$ \\
Mediana & $4,37 \mathrm{bA}$ & $6,37 \mathrm{aA}$ & & $0,35 \mathrm{bA}$ & $0,38 \mathrm{aA}$ \\
Basal & $4,20 \mathrm{~A}$ & $6,60 \mathrm{~A}$ & & $0,28 \mathrm{bB}$ & $0,38 \mathrm{aA}$ \\
\hline
\end{tabular}

Letras minúsculas comparam as médias dentro da linha e as maiúsculas dentro da coluna para cada característica (lowercase letters compare the averages within the line and capital letters within the column for each characteristic).

Tabela 4. Porcentagem de enraizamento e de sobrevivência de estacas de Turnera subulata obtidas em diferentes posições no ramo da planta e com ausência ou presença de folhas (rooting percentage and survival of cuttings of Turnera subulata obtained at different positions in the plant and with presence or absence of leaves). Redenção, UNILAB, 2013.

\begin{tabular}{lccccc}
\hline \multirow{2}{*}{ Tipo de Estaca } & \multicolumn{2}{c}{ Enraizamento (\%) } & & \multicolumn{2}{c}{ Sobrevivência (\%) } \\
\cline { 2 - 3 } \cline { 5 - 6 } & Ausência de folhas & Presença de folhas & & Ausência de folhas & Presença de folhas \\
\hline Apical & $70,78 \mathrm{aA}$ & $67,45 \mathrm{aB}$ & & $78 \mathrm{aA}$ & $70 \mathrm{aB}$ \\
Mediana & $77,90 \mathrm{aA}$ & $98,80 \mathrm{aA}$ & & $70 \mathrm{aA}$ & $100 \mathrm{aA}$ \\
Basal & $78,47 \mathrm{bA}$ & $96,85 \mathrm{aA}$ & & $70 \mathrm{bA}$ & $100 \mathrm{aA}$ \\
\hline
\end{tabular}

Letras minúsculas comparam as médias dentro da linha e as maiúsculas dentro da coluna para cada característica (lowercase letters compare the averages within the line and capital letters within the column for each characteristic).

das raízes. Salomão et al. (2002) verificaram que estacas de maracujazeiros doce (Passiflora alata) e amarelo ( $P$. edulis f. flavicarpa) das posições basal e mediana apresentaram maior potencial de enraizamento e maior crescimento do sistema radicular. Segundo Purcino et al . (2012), a ausência de folhas em estacas de alfavaca-cravo (O. gratissimum) reduziu drasticamente a porcentagem de estacas enraizadas apresentando alta mortalidade das estacas (30\%).

Por outro lado, resultados contrários aos do presente estudo foram encontrados por outros autores, como Tavares et al. (2012) que verificaram em três quimiotipos de cidreira (Lipia alba) que tanto estacas apicais como basais podem ser utilizadas na propagação vegetativa;
Garbuio et al. (2007) observaram que na propagação vegetativa do patchouli (Pogostemon cablin), a porcentagem de brotação, enraizamento, comprimento médio das três maiores raízes e número de raízes emitidas por estacas decresceu das estacas apicais para as basais.

Paulino et al. (2011), em estudo de propagação por estacas de $T$. subulata em diferentes substratos e posição de obtenção da estaca nos ramos concluíram que os melhores tipos de estacas para enraizamento são as medianas e basais em substrato areia ou arisco. Portanto, o presente estudo confirma que a escolha da região do ramo para produção de estacas de Turnera subulata é um fator importante no enraizamento dessa espécie. Além disso, a presença de folhas é vital na sua propagação, destacando as estacas basais. Portanto, recomenda-se o uso de estacas basais com duas folhas para a propagação de Turnera subulata.

\section{REFERÊNCIAS}

AGRA, MF; FREITAS, PF; BARBOSA-FILHO, JM. 2007. Synopsis of the plants known as medicinal and poisonous in northeast of Brazil. Revista Brasileira de Farmacognosia 17: 114-140.

ALBUQUERQUE JUNIOR, CL; DANNER, MA; KANIS, LA; DESCHAMPS, C; ZANETTE, F; FARIAS, PM. 2013. Enraizamento de estacas semilenhosas de maracujazeiro amarelo (Passiflora actínia Hook). Semina: 34: 3663-3668.

ALBUQUERQUE, UP; MEDEIROS, PM; ALMEIDA, ALS; MONTEIRO, JM; LINS 
NETO, EMF; MELO, JG; SANTOS, JP. 2007. Medicinal plants of the caatinga (semiarid) vegetation of NE Brazil: A quantitative approach. Journal of Ethnopharmacology 114: 325-354.

ANTOUN, MD; RAMOS, Z; VASQUEZ, J; OQUENDO, I; PROCTOR, GR; GERENA, L; FRANZBLAU, SG. 2001. Evaluation of the flora of Puerto Rico for in vitro antiplasmodial and antimycobacterial activities. Phytotherapy Research 15: 638-642.

ARBO, MM. 2005. Estudios sistemáticos em Turnera (Turneraceae). III Series Anomalae y Turnera. Bonplandia 14: 115-318.

CARVALHO, JSB; NUNES, MFPN; CAMPOS, GPA; GOES, MCC. 2015. Influência de diferentes tipos de estacas e substratos na propagação vegetativa de Hyptis pectinata. Revista de Ciências Agroveterinárias 14: 89-91.

GALVEZ, J. 2006. Intestinal antiinflammatory activity of a lyophilized infusion of Turnera ulmifolia in TNBS rat colitis. Fitoterapia 77: 515-520.

GARBUIO, C; BIASI, LA; KOWALSKI, APJ; SIGNOR, D; MACHADO, EM; DESCHAMPS, C. 2007. Propagação por estaquia em patchouli com diferentes números de folhas e tipos de estacas. Scientia Agraria 8: 435-438.

HARTMANN, HT; KESTER, DE; DAVIES,
FT; GENEVE, R. 2011. Plant propagation: principles and practices. New Jersey: Prentice Hall, 915p.

NASCIMENTO, MA; SILVA, AK; FRANCCA, LC; QUIGNARD, EL; LÓPEZ, JA; ALMEIDA, MG. 2006. Turnera ulmifolia L. (Turneraceae): Preliminary study of its antioxidant activity. Bioresources Technology 97: 1387-1391.

OLIVEIRA, AF; PASQUAL, M; CHALFUN, NNJ; REGINA, MA; RINCÓN, CR. 2003. Influência do número de nós em estacas semilenhosas de oliveira (Olea europaea L.) no enraizamento sob câmara de nebulização. Ciência agrotecnica 27:332-338.

PAULINO, RC; HENRIQUES, GPSA; MAIA, SSS; COELHO, MFB. 2011. Diferentes tipos de estacas e de substratos na propagação vegetativa de xanana (Turnera subulata $\mathrm{Sm}$.). Revista Verde 6: 234-237.

PIO, R; CHALFUN, NNJ; RAMOS, JD; GONTIJO, TCA; TOLEDO, M; CARRIJO, EP. 2004. Presença de folhas e gema apical no enraizamento de estacas herbáceas de figueira oriundas da desbrota. Revista Brasileira de Agrociência10: 51-54.

PURCINO, M; MACHADO, MP; BIASI, LA. 2012. Efeito das folhas no enraizamento de estacas de alfavaca-cravo e alfavaca-anis. Revista de Ciências Agroveterinárias 11: 93-98.

SALOMÃO, LCC; PEREIRA, WE; DUARTE,
RCC; SIQUEIRA, DL. 2002. Propagação por estaquia dos maracujazeiros doce (Passiflora alata Dryand.) e amarelo (P. edulis f. Flavicarpa O. Deg.). Revista Brasileira de Fruticultura 24: 163-167.

SANTOS, NC; DIAS, CN; MORAES, DFC; VILANOVA, CM; GONÇALVES, JRS; SOUZA, NS; ROSA, IG. 2010. Toxicidade e avaliação de atividade moluscicida de folhas de Turnera ulmifolia L. Revista Brasileira de Biociências 8: 324-329.

SCHLINDWEIN, C; MEDEIROS, PCR. 2006. Pollination in Turnera subulata (Turneraceae): Unilateral reproductive dependence of the narrowly oligolectic bee Protomeliturga turnerae (Hymenoptera, Andrenidae). Flora 201: 178-188.

SHORE, JS; ARBO, MM; FERNÁNDEZ, A. 2006. Breeding system variation, genetics and evolution in the Turneraceae. New Phytologist 171: 539-551.

TAVARES, IB; MOMENTÉ, VG; BARRETO, HG; CASTRO, HG; SANTOS, GR; NASCIMENTO, IR. 2012. Tipos de estacas e diferentes substratos na propagação vegetativa da erva cidreira (quimiotipos I, II E III). Bioscience Journal 28: 206-213.

VIGNOLO,GK; PICOLOTTO, L; GONÇALVES, MA; PEREIRA, IS; ANTUNES, LEC. 2014. Presença de folhas no enraizamento de estacas de amoreira-preta. Ciência Rural 44: 467-472. 